\title{
Sildenafil in a cigarette smoke-induced model of COPD in the guinea-pig
}

\author{
David Domínguez-Fandos ${ }^{1}$, César Valdés ${ }^{1}$, Elisabet Ferrer ${ }^{1}$, Raquel Puig-Pey ${ }^{1}$, \\ Isabel Blanco ${ }^{1,2}$, Olga Tura-Ceide ${ }^{1,2}$, Tanja Paul ${ }^{1}$, Víctor I. Peinado ${ }^{1,2}$ and
} Joan A. Barberà ${ }^{1,2}$

Affiliations: ${ }^{1}$ Dept of Pulmonary Medicine, Hospital Clínic-Institut d'Investigacions Biomèdiques August Pi i Sunyer (IDIBAPS), University of Barcelona, Barcelona, Spain. ${ }^{2}$ Centro de Investigación Biomédica en Red (CIBER) de Enfermedades Respiratorias, Madrid, Spain.

Correspondence: Joan A. Barberà, Servei de Pneumologia, Hospital Clínic, Villarroel 170, 08036 Barcelona, Spain. E-mail: jbarberalaclinic.ub.es

ABSTRACT Sildenafil, a phosphodiesterase-5 inhibitor used to treat pulmonary hypertension, may have effects on pulmonary vessel structure and function. We evaluated the effects of sildenafil in a cigarette smoke (CS)-exposed model of chronic obstructive pulmonary disease (COPD).

42 guinea-pigs were exposed to cigarette smoke or sham-exposed and treated with sildenafil or vehicle for 12 weeks, divided into four groups. Assessments included respiratory resistance, pulmonary artery pressure (PAP), right ventricle (RV) hypertrophy, endothelial function of the pulmonary artery and lung vessel and parenchymal morphometry.

CS-exposed animals showed increased PAP, RV hypertrophy, raised respiratory resistance, airspace enlargement and intrapulmonary vessel remodelling. CS exposure also produced wall thickening, increased contractility and endothelial dysfunction in the main pulmonary artery. CS-exposed animals treated with sildenafil showed lower PAP and a trend to less RV hypertrophy than CS-exposed only animals. Furthermore, sildenafil preserved the intrapulmonary vessel density and attenuated the airspace enlargement induced by CS. No differences in gas exchange, respiratory resistance, endothelial function and vessel remodelling were observed.

We conclude that in this experimental model of COPD, sildenafil prevents the development of pulmonary hypertension and contributes to preserve the parenchymal and vascular integrity, reinforcing the notion that the nitric oxide-cyclic guanosine monophosphate axis is perturbed by CS exposure.

@ERSpublications

Sildenafil reduces pulmonary vascular tone and contributes to preserve tissue integrity in experimental COPD http://ow.ly/KAr6k

This article has supplementary material available from erj.ersjournals.com

Received: July 312014 | Accepted after revision: March 052015 | First published online: April 302015

Support statement: Supported by grants FIS PI09/00536 and PI13/00836 and R5-HCPB. Funding information for this article has been deposited with FundRef.

Conflict of interest: Disclosures can be found alongside the online version of this article at erj.ersjournals.com

Copyright OERS 2015 


\section{Introduction}

Pulmonary hypertension $(\mathrm{PH})$ is a frequent and serious complication of chronic obstructive pulmonary disease (COPD), triggered in part by cigarette smoke (CS) exposure [1]. The pathophysiology of $\mathrm{PH}$ in COPD involves endothelial dysfunction, imbalance of growth factors [2] and an enhanced inflammatory response [3] in pulmonary vessels. These factors, alone or in combination, induce smooth muscle cell proliferation in the vessel wall, leading to increased pulmonary vascular resistance.

Endothelial dysfunction in the pulmonary arteries of COPD patients is associated with reduced endothelial nitric oxide synthase (eNOS) expression and impaired release of nitric oxide $[4,5]$. Endothelial nitric oxide activates the soluble guanylate cyclase (sGC), resulting in the formation of the secondary messenger cyclic guanosine monophosphate (cGMP) [4, 6]. Intracellular cGMP decreases the concentration of intracellular calcium, thereby relaxing vascular smooth muscle cells [7]. Endothelial nitric oxide may also inhibit smooth muscle cell proliferation through cGMP-dependent mechanisms [8]. In the lung, cGMP is metabolised primarily by the action of phosphodiesterase (PDE)5. Inhibitors of PDE5, such as sildenafil, enhance the nitric oxide-cGMP signalling pathway and exert vasodilator and antiproliferative effects [9]. Studies in experimental models of PH induced by hypoxia [10] or monocrotaline $[11,12]$ have shown that sildenafil reduces pulmonary artery pressure (PAP), prevents right ventricular (RV) hypertrophy and exerts an anti-remodelling effect in pulmonary vessels. Sildenafil is currently used for the treatment of pulmonary arterial hypertension [13].

In patients with COPD and associated $\mathrm{PH}$, we have demonstrated that sildenafil decreases pulmonary vascular resistance acutely [14], but this effect did not translate into augmented exercise tolerance when administered over 3 months [15]. This limited influence on exercise tolerance could be due to the concomitant changes that occur in the lung parenchyma and airways of COPD patients.

The effects of sildenafil on lung structure have not been evaluated in experimental models of COPD. The utilisation of guinea-pigs exposed to CS is the most advantageous approach to reproduce COPD features by using the primary disease-causing agent [16, 17]. This species lacks hypoxic pulmonary vasoconstriction (HPV), but develops chronic PH after exposure to CS for relatively long periods [17]. The lack of HPV is an additional advantage in testing the anti-remodelling effects of vasodilators, minimising a potential detrimental effect on gas exchange.

In a recent study we demonstrated that the administration of a nitric oxide-independent sGC stimulator to guinea-pigs chronically exposed to CS reduces pulmonary vascular resistance and prevents pulmonary vascular remodelling, as well as the development of emphysema [18]. Therefore, we hypothesised that sildenafil, which enhances the activity of cGMP by impeding its metabolisation, might exert favourable effects on lung structure beyond its vasodilator action.

Accordingly, the present study aimed to evaluate the effects of sildenafil on pulmonary haemodynamics, endothelial function and vascular and parenchymal remodelling in guinea-pigs chronically exposed to CS.

\section{Methods \\ Experimental groups}

42 male guinea-pigs were divided into two groups: exposed to the smoke of seven nonfiltered research cigarettes per day (3R4F; Kentucky University Research, Lexington, KY, USA) (smoke content per cigarette $11 \mathrm{mg}$ total particulate matter, $9.4 \mathrm{mg}$ tar, $0.73 \mathrm{mg}$ nicotine and $12 \mathrm{mg}$ carbon monoxide), 5 days per week, for 12 weeks $(n=26)$; and sham-exposed $(n=16)$, using a nose-only system (Protowerx Design Inc, Langley, BC, Canada). Daily, after CS or sham exposure, animals were administered with a vehicle (distilled water $\mathrm{n}=18$ ), or treated with $1 \mathrm{mg} \cdot \mathrm{kg}^{-1}$ of sildenafil citrate solution by gavage $(\mathrm{n}=24)$, resulting in four experimental groups. Sildenafil (UK-92,480-10) was kindly provided by Pfizer (Sandwich, UK).

All animal procedures were approved by the ethics review board on animal research of the University of Barcelona and complied with national and international guidelines.

\section{Unrestrained whole-body plethysmography}

Respiratory function was measured weekly by unrestrained whole-body plethysmography (Buxco Research Systems, Wilmington, NC, USA) as previously described [19]. Breathing frequency, tidal volume $(V \mathrm{~T})$, minute ventilation $\left(V^{\prime} \mathrm{E}\right)$ and respiratory resistance (enhanced pause (Penh)) [20] were recorded $10 \mathrm{~min}$ before exposure (baseline) and $10 \mathrm{~min}$ after CS or sham exposure.

\section{Pulmonary haemodynamics and arterial oxygenation}

At the end of the experimental protocol and $24 \mathrm{~h}$ after the last exposure to CS and sildenafil dose, systolic $(\mathrm{s})$, diastolic (d) and mean (m)PAP were measured under anaesthesia in open-chest animals using a 
catheter placed in the main pulmonary artery through the RV and connected to a pressure transducer (Buxco Research Systems).

Arterial oxygen tension $\left(\mathrm{PaO}_{2}\right)$ was analysed in blood sampled from the carotid artery, immediately after haemodynamic measurements. The animals were subsequently sacrificed.

\section{Right ventricle hypertrophy}

The heart was removed and RV and left ventricle plus the septum $(\mathrm{LV}+\mathrm{S})$ were dissected and weighed separately, and the ratio between $\mathrm{RV}$ and $\mathrm{LV}+\mathrm{S}$ weights $(\mathrm{RV} / \mathrm{LV}+\mathrm{S})$ was calculated.

\section{Endothelial function}

The main pulmonary artery was isolated, cleaned of fat and connective tissue and cut into rings $3 \mathrm{~mm}$ long. The left branch was placed in an organ bath chamber and attached to an isometric transducer (Panlab, Barcelona, Spain). After a period of stabilisation, arteries were contracted using potassium chloride $(\mathrm{KCl})(60 \mathrm{mM})$ to determine their viability and contractile capacity. Endothelial function was assessed in pre-contracted pulmonary artery rings, as previously described [17], by measuring changes in wall tension in response to cumulative doses of adenosine diphosphate (ADP). At the end of the studies, main pulmonary artery rings were fixed and cryo-embedded for histological examination.

\section{Morphometric and histological assessments}

Explanted lungs were inflated and fixed with formalin under a constant pressure of $30 \mathrm{cmH}_{2} \mathrm{O}$ for $24 \mathrm{~h}$ and then embedded in paraffin.

The wall thickness of the main pulmonary artery was measured in sections stained with elastin-Van Gieson. Vascular density was assessed as the number of pulmonary vessels per $\mathrm{mm}^{2}$ of lung tissue. The number of small intrapulmonary vessels (diameter $<50 \mu \mathrm{m}$ ) showing positive immunostaining for smooth muscle $\alpha$-actin or with double elastic laminae in orcein-stained sections were counted, and expressed as a percentage of the total number of small intrapulmonary vessels. Intrapulmonary vessels immunostained for $\alpha$-actin were further classified semi-quantitatively, depending on the proportion of the vessel wall positive for $\alpha$-actin: nonmuscularised $\leqslant 1 / 4$ of the vessel wall; partially muscularised $>1 / 4$ to $\leqslant 3 / 4$ of the vessel wall; or fully muscularised $>3 / 4$ of the muscle wall.

The mean airspace size was evaluated in haematoxylin-stained tissue sections by measuring the mean linear intercept (MLI) of alveolar septa in 20 randomly selected fields.

\section{Real-time PCR}

Total RNA was extracted from lung tissue using the RNeasy Micro Kit (Qiagen GmbH, Hilden, Germany). Total RNA was retrotranscribed and quantification of eNOS was performed by real-time PCR, as previously described [17]. Expression of eNOS was normalised to $\beta$-actin expression, as endogenous housekeeping gene and relative gene expression was analysed using the $2^{-\Delta \Delta \mathrm{C}_{\mathrm{T}}}$ method [21].

\section{Data analysis}

Results are expressed as mean \pm SD or as median and interquartile range, depending on whether or not the variables followed a normal distribution. The progression of respiratory parameters (Penh, breathing frequency, $V \mathrm{~T}$ and $V^{\prime} \mathrm{E}$ ) was assessed weekly throughout the 3 months of the study and expressed as the area under the curve (AUC) of all measurements.

Comparisons between groups were performed using a two-way ANOVA, considering exposure to CS and sildenafil as main factors, and their interaction. When significant, post hoc pairwise comparisons were performed using the Student-Newman-Keuls test. Relationships between variables were assessed using Pearson's correlation test. A p-value $<0.05$ was considered to be significant.

\section{Results}

Pulmonary haemodynamics and right ventricle hypertrophy

Animals exposed to CS showed higher PAP than nonexposed animals ( $p=0.005)$ (fig. 1a and table 1). Treatment with sildenafil prevented the increase of PAP induced by CS, and PAP values were similar to sham-exposed animals (fig. 1a and table 1).

Untreated CS-exposed animals showed RV hypertrophy. In guinea-pigs exposed to CS and treated with sildenafil the RV/LV+S ratio was lower than in the CS-exposed group, although not significantly, and not different from sham-exposed animals (fig. 1b). 

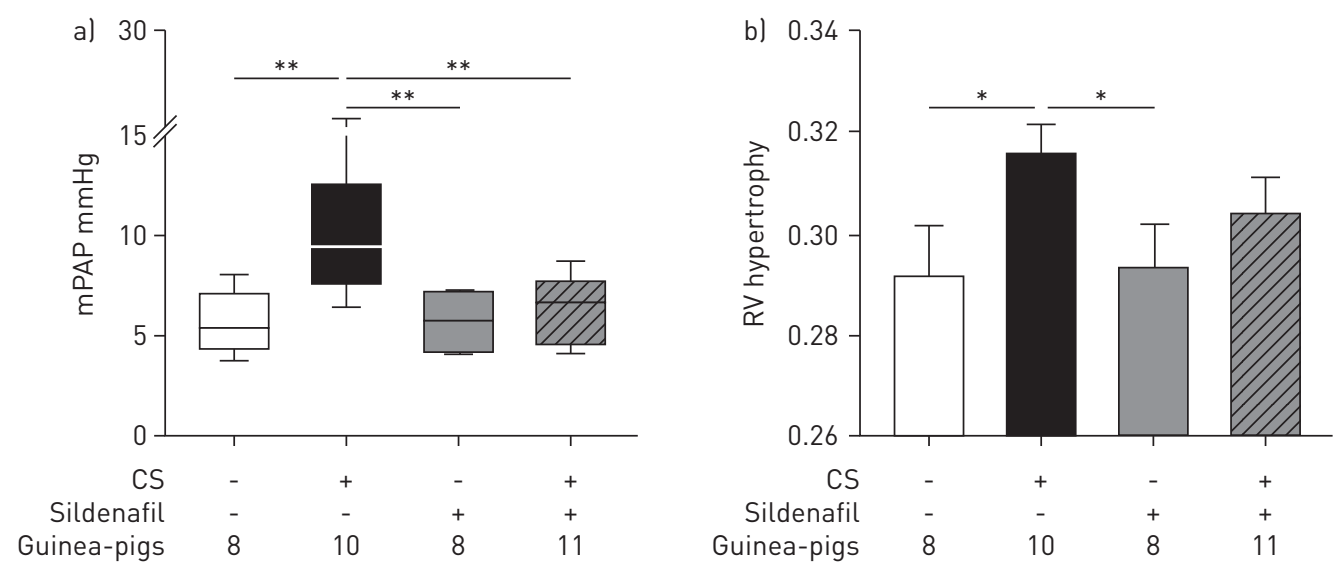

FIGURE 1 Pulmonary artery pressure and right ventricle hypertrophy. a) Mean pulmonary artery pressure (mPAP). Data are presented as median (interquartile range); the whiskers represent the 90th and 10th percentiles. b) Right ventricle (RV) hypertrophy assessed by the weight ratio of the RV and the left ventricle plus septum. Data are presented as mean \pm SEM. Treatment with sildenafil in cigarette smoke (CS)-exposed animals prevented the increase in mPAP and attenuated RV hypertrophy. ${ }^{*}$ : $\mathrm{p}<0.05 ;{ }^{* *}$ : $\mathrm{p}<0.01$.

\section{Endothelial function and vascular contractility}

The maximal contraction induced by $\mathrm{KCl}$ in pulmonary arteries was greater in animals exposed to CS (table 1). Sildenafil treatment did not modify the contractile response of pulmonary arteries.

Endothelium-dependent relaxation induced by cumulative doses of ADP was slightly attenuated in animals exposed to CS, as suggested by a trend to a right shift $(\mathrm{p}=0.096)$ and a higher AUC $(\mathrm{p}=0.058)$ of the dose-response curve (table 1 and online supplementary fig. S1). Concomitant treatment with sildenafil did not modify the endothelium-dependent vasodilator response.

\section{Morphometric and histological assessments}

Wall thickness of the main pulmonary artery was greater in animals exposed to CS (supplementary fig. S2). No difference was observed in animals exposed to CS and treated with sildenafil (table 2).

The proportion of small vessels showing positive immunoreactivity to smooth muscle $\alpha$-actin was higher in animals exposed to CS (fig. 2 and table 2). When vessels were scored according to the degree of muscularisation, it was apparent that in the CS-exposed groups there was a decrease in the proportion of

\section{TABLE 1 Assessment of in vivo pulmonary artery pressure and in vitro reactivity}

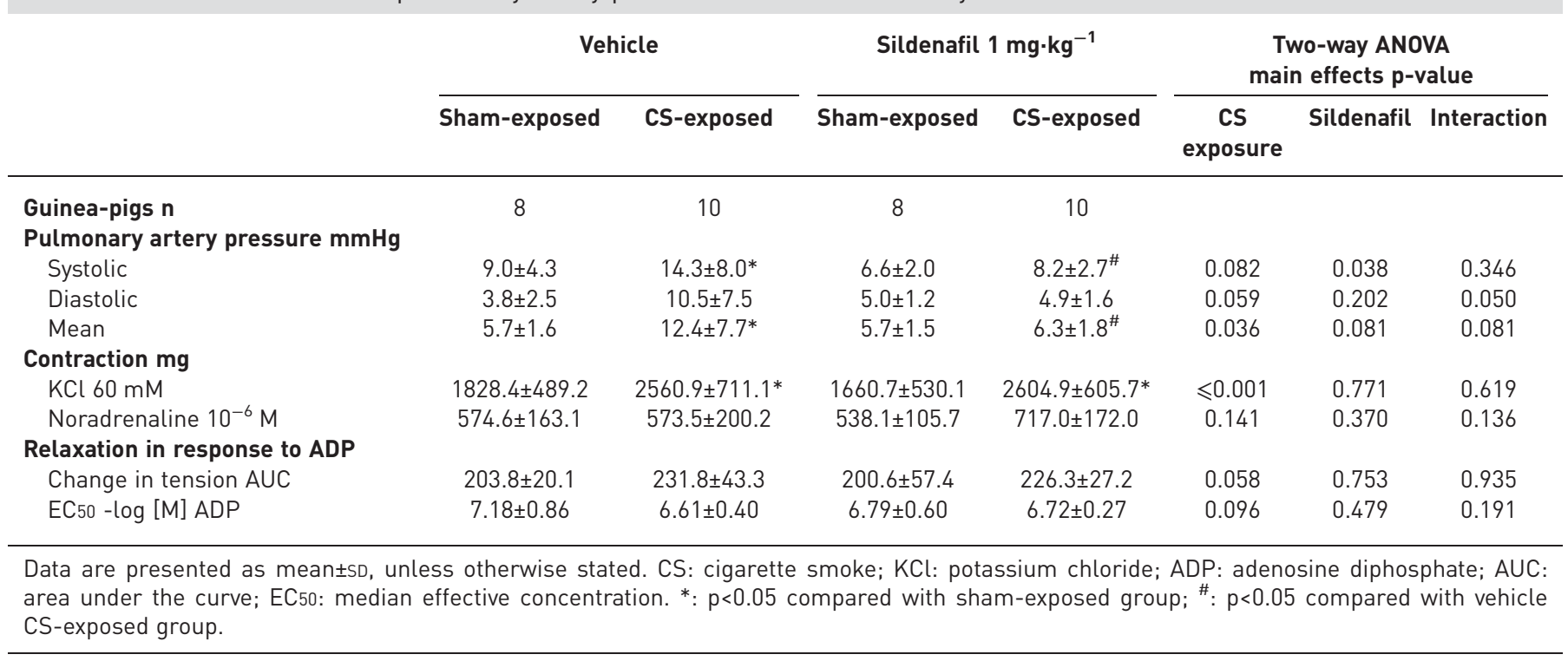


TABLE 2 Morphological assessments and quantification of endothelial nitric oxide synthase (eNOS) expression

Vehicle

Sildenafil

Two-way ANOVA

main effects $p$-value

\section{Sham-exposed CS-exposed Sham-exposed CS-exposed \\ CS Sildenafil Interaction} exposure

\begin{tabular}{|c|c|c|c|c|c|c|c|}
\hline Guinea-pigs n & 8 & 10 & 8 & 11 & & & \\
\hline Pulmonary artery wall thickness $\mu \mathrm{m}$ & $57.7 \pm 23.3$ & $77.8 \pm 9.0^{*}$ & $54.8 \pm 4.7$ & $75.4 \pm 9.8^{*}$ & $\leqslant 0.001$ & 0.565 & 0.963 \\
\hline Smooth muscle $\alpha$-actin-positive arteries \% & $25.1 \pm 5.5$ & $40.3 \pm 13.1^{*}$ & $26.4 \pm 4.6$ & $39.8 \pm 7.1^{*}$ & $\leqslant 0.001$ & 0.877 & 0.760 \\
\hline Arteries with double elastic lamina \% & $1.3 \pm 2.5$ & $4.1 \pm 6.6$ & $0.7 \pm 2.0$ & $2.8 \pm 1.2$ & 0.101 & 0.512 & 0.833 \\
\hline Vessels $<50 \mu \mathrm{m}$ per $\mathrm{mm}^{-2}$ & $23.5 \pm 4.2$ & $19.0 \pm 5.6$ & $27.3 \pm 5.0$ & $25.4 \pm 5.7^{\#}$ & 0.077 & 0.006 & 0.476 \\
\hline Interseptal distance $\mu \mathrm{m}$ & $69.1 \pm 12.8$ & $78.0 \pm 11.8$ & $64.7 \pm 7.6$ & $71.3 \pm 7.9$ & 0.028 & 0.114 & 0.749 \\
\hline Relative eNOS expression eNOS/ $\beta$-actin & $1.07 \pm 0.14$ & $1.08 \pm 0.13$ & $1.10 \pm 0.14$ & $0.96 \pm 0.12$ & 0.632 & 0.699 & 0.586 \\
\hline
\end{tabular}

Data are presented as mean $\pm \mathrm{SD}$, unless otherwise stated. CS: cigarette smoke. ${ }^{*}: \mathrm{p}<0.05$ compared with sham-exposed group; ${ }^{*}: \mathrm{p}<0.01$ compared with vehicle CS-exposed group.
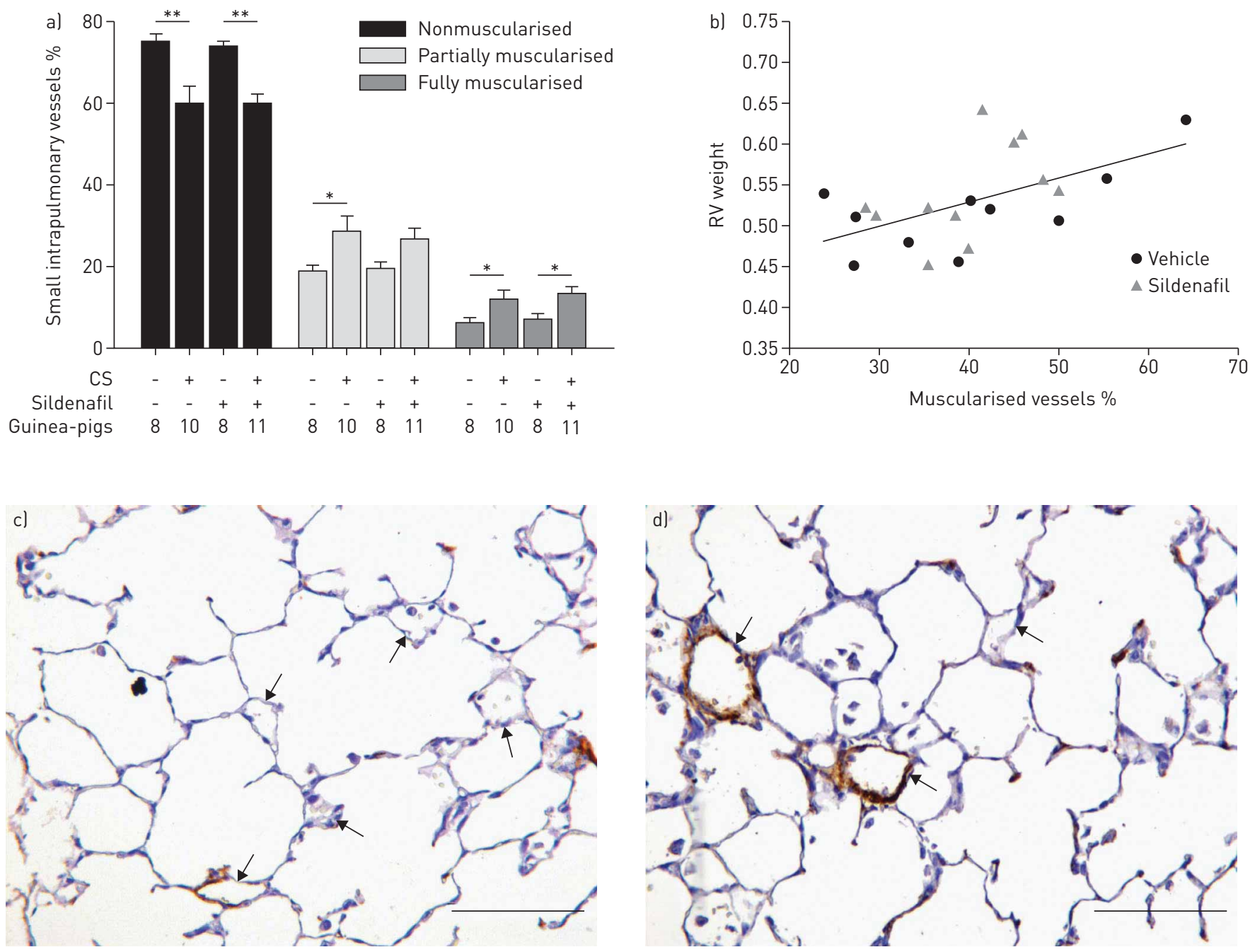

FIGURE 2 Muscularisation of small intrapulmonary arteries $(<50 \mu \mathrm{m})$. a) Percentage of small arteries according to their degree of muscularisation. Animals exposed to cigarette smoke (CS), irrespective of sildenafil administration, showed a lower number of nonmuscularised arteries and developed a greater number of partially and fully muscularised arteries. Data are presented as mean \pm SEM. ${ }^{*}: \mathrm{p}<0.05 ;{ }^{* *}: \mathrm{p}<0.01 . \mathrm{b}$ ) Correlation between the percentage of muscularised small intrapulmonary vessels and the weight of the right ventricle (RV) in guinea-pigs exposed to CS treated with vehicle or sildenafil; $r=0.55$, $p=0.01$. Photomicrographs of $\alpha$-actin immunostained small pulmonary vessels in c) a control guinea-pig and d) an animal exposed to CS. In the exposed animal, fully muscularised arteries are present. Arrows indicate small intrapulmonary vessels. Scale bars $=100 \mu \mathrm{m}$. 
nonmuscularised vessels and a concomitant increase in the proportion of partially and fully muscularised intrapulmonary vessels (fig. 2). Treatment with sildenafil did not modify changes induced by CS.

No significant differences between groups were observed in the proportion of arteries with double elastic laminae (table 2).

Exposure to CS showed a trend to reduce the density of small intrapulmonary vessels. Animals treated with sildenafil showed greater small vessel density, thereby preventing the reduction induced by CS exposure ( $\mathrm{p}=0.009)$ (fig. 3 and table 2).

Development of pulmonary emphysema, assessed as an increased MLI, was observed in animals exposed to CS compared with the sham-exposed group ( $\mathrm{p}=0.028$ ) (fig. 4 and table 2). Guinea-pigs exposed to CS and treated with sildenafil showed intermediate MLI values that did not differ either from CS-exposed or from sham-exposed animals (fig. 4 and table 2).

\section{Gene expression of eNOS}

No differences in the gene expression of eNOS in lung homogenates were observed between groups (table 2).

\section{Pulmonary function and blood gas measurements}

Unrestrained whole-body plethysmographic measurements performed before CS or sham exposure showed a trend to lower $V^{\prime} \mathrm{E}(\mathrm{p}=0.051)$ (supplementary table S1). After CS exposure a dramatic increase in $V \mathrm{~T}, V^{\prime} \mathrm{E}$ and Penh occurred (supplementary fig. S3b and supplementary table S1). The concomitant administration of sildenafil neither modified the pulmonary function at baseline nor after CS exposure (supplementary fig. S3a and supplementary table S1).

Guinea-pigs exposed to CS showed a trend to lower $\mathrm{PaO}_{2}$ than nonexposed animals $(\mathrm{p}=0.138)$. The administration of sildenafil did not modify the $\mathrm{PaO}_{2}$ value in CS-exposed nor in nonexposed guinea-pigs (supplementary table S1).

\section{Correlations}

In CS-exposed animals, the muscularisation of small pulmonary vessels correlated with the RV weight $(\mathrm{r}=0.55, \mathrm{p}=0.01)$ (fig. $2 \mathrm{~b})$ and the density of small intrapulmonary vessels was inversely related to RV hypertrophy $(\mathrm{r}=-0.52, \mathrm{p}=0.01$ ) (fig. $3 \mathrm{~b})$. Furthermore, the MLI correlated with mPAP $(\mathrm{r}=0.56, \mathrm{p}=0.01)$ and with the total number of small pulmonary vessels ( $r=-0.44, p=0.01$ ) (fig. $4 b$ ).

\section{Discussion}

The present study evaluated the effects of sildenafil in guinea-pigs chronically exposed to CS. Our results show that sildenafil prevented the increase in PAP and the subsequent RV hypertrophy induced by CS, but did not modify pulmonary vascular remodelling. Furthermore, sildenafil showed a trend to diminish the airspace enlargement induced by CS exposure and preserved the intrapulmonary vessel density.
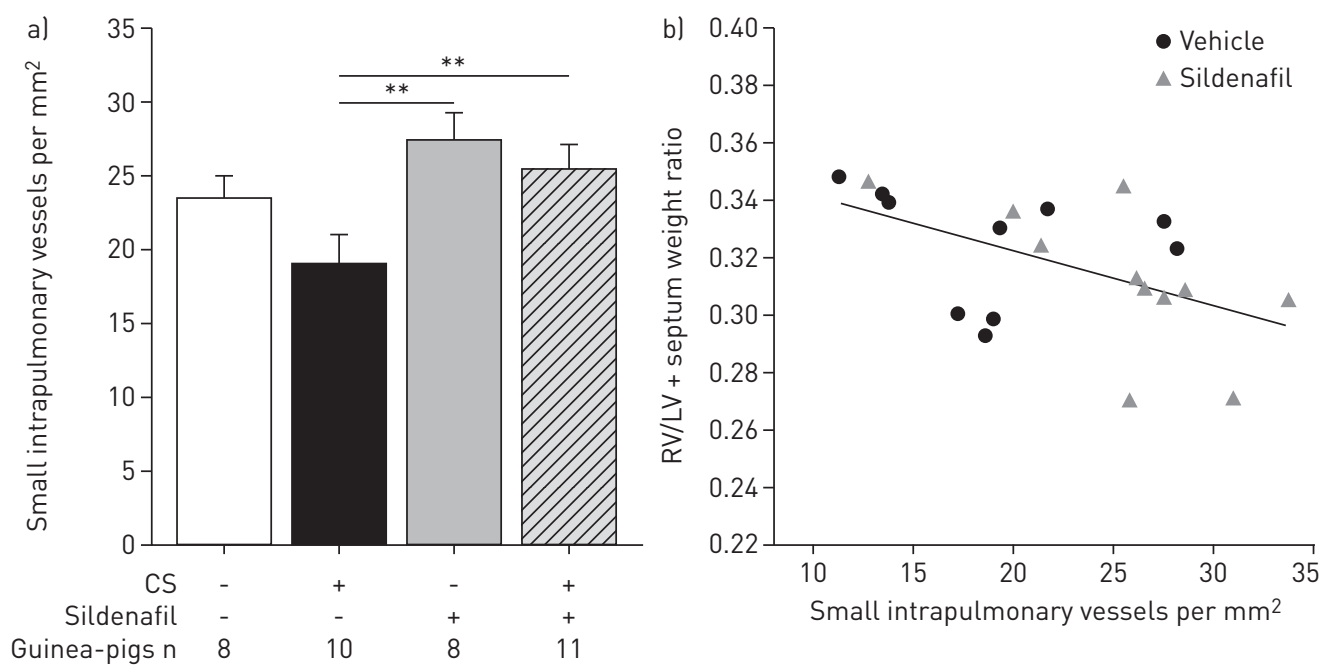

FIGURE 3 Density of small intrapulmonary vessels $(<50 \mu \mathrm{m})$. a) Cigarette smoke (CS)-exposed animals treated with sildenafil showed a greater density of small intrapulmonary vessels than CS-exposed only. Data are presented as mean \pm SEM. **: $\mathrm{p}<0.01$. b) Correlation between the density of small intrapulmonary vessels and right ventricular hypertrophy (RV/left ventricle (LV)+septum weight ratio) in guinea-pigs exposed to CS treated with vehicle or sildenafil; $r=-0.52, p=0.01$. 

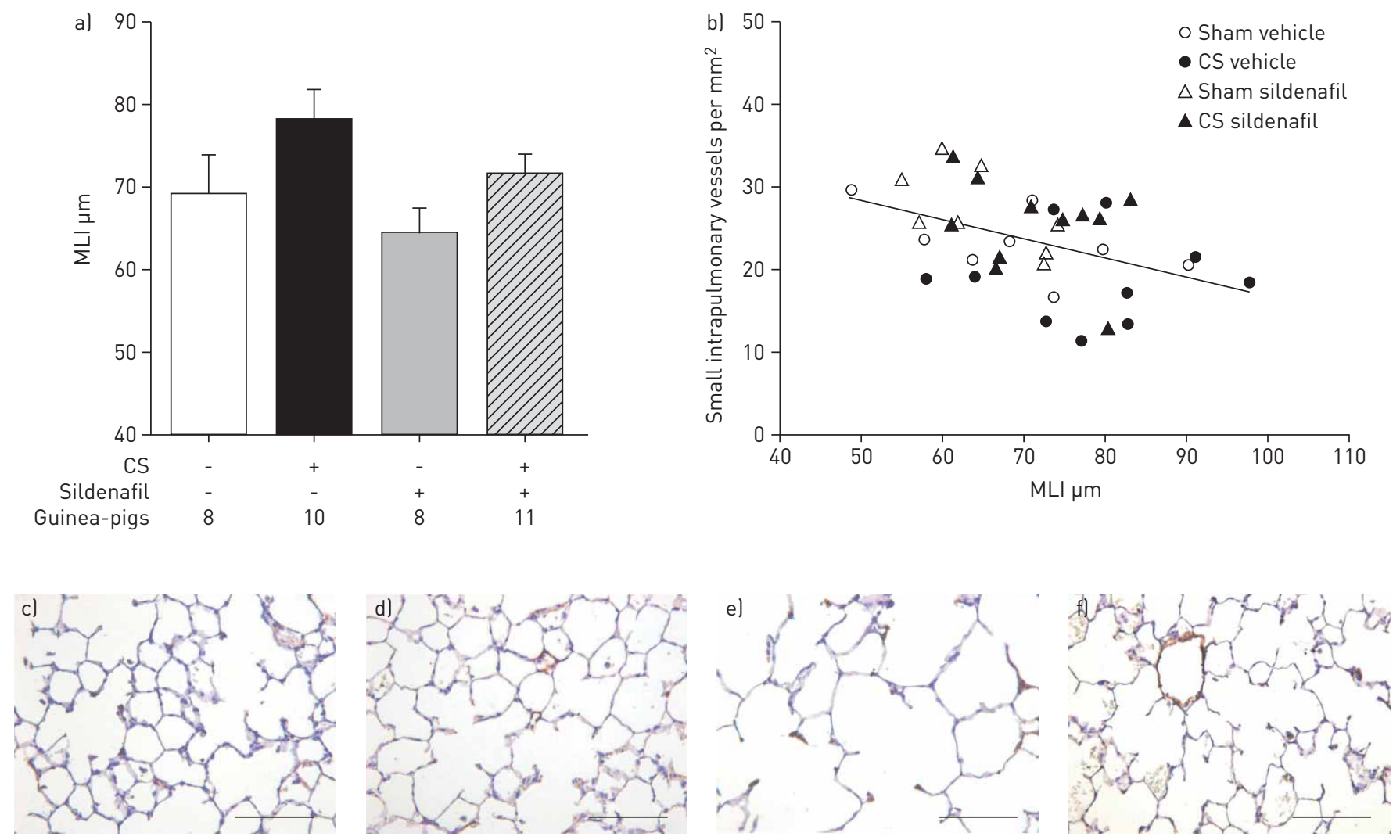

FIGURE 4 a) Airspace size, evaluated by the mean linear intercept (MLI). Main effects in the two-way ANOVA: cigarette smoke (CS) p=0.028 and sildenafil $\mathrm{p}=0.114$. Data are presented as mean \pm SEM. b) Correlation between the MLI and the pulmonary vascular density (number of vessels $<50 \mu \mathrm{m}$ per $\mathrm{mm}^{2}$ ); $\mathrm{r}=0.44$, $\mathrm{p}=0.01$. Photomicrographs of lung parenchyma sections stained with haematoxylin in sham-exposed guinea-pigs treated with $\mathrm{c}$ ) vehicle or $\mathrm{d}$ ) sildenafil; and CS-exposed guinea-pigs treated with e) vehicle or f) sildenafil. Scale bars $=100 \mu \mathrm{m}$.

The main effect observed in this study was the prevention of PH in CS-exposed animals treated with sildenafil. Whereas guinea-pigs exposed to CS showed increased mPAP and RV hypertrophy compared with nonexposed animals, in keeping with previous studies [22, 23]; CS-exposed, sildenafil-treated animals displayed mPAP values and a $\mathrm{RV} / \mathrm{LV}+\mathrm{S}$ weight ratio similar to the nonexposed animals, confirming the vasodilator action of sildenafil in this experimental model [10-12]. The lack of increase in PAP was not accompanied by differences in lung vessel structure, since CS-exposed guinea-pigs treated with sildenafil showed pulmonary artery wall thickening, muscularisation of small vessels and proliferation of smooth muscle cells at similar levels to CS-exposed, untreated guinea-pigs and at significantly higher levels than in nonexposed animals. Therefore, in the current experimental setting sildenafil exerted a vasodilator, but not anti-remodelling effect, on pulmonary arteries.

Previous studies in experimental models of $\mathrm{PH}$ induced by hypoxia have provided discrepant results regarding the anti-remodelling effect of sildenafil in pulmonary vessels. Whereas in some studies sildenafil prevented pulmonary vascular remodelling, along with a reduction of PAP [10, 24], in others it failed to exert an anti-remodelling effect [25], despite reducing PAP, similar to our study. We used a sildenafil dose similar to that approved in humans with pulmonary arterial hypertension [26] $\left(1 \mathrm{mg} \cdot \mathrm{kg}^{-1}\right.$ per day over 12 weeks; cumulative dose $84 \mathrm{mg} \cdot \mathrm{kg}^{-1}$ ), which is a low dose compared with previous studies in experimental models of $\mathrm{PH}$ induced by hypoxia [10, 24, 25, 27]. It is tempting to speculate that such a low dose could have been sufficient to exert vasodilation, but insufficient to produce an anti-remodelling effect. Nevertheless, in previous studies in experimental $\mathrm{PH}$, a significant anti-remodelling effect has been shown with cumulative sildenafil doses of $180 \mathrm{mg} \cdot \mathrm{kg}^{-1}$ [24], whereas cumulative doses of $525 \mathrm{mg} \cdot \mathrm{kg}^{-1}$ failed to produce any anti-remodelling effect [25]. We cannot disregard the suggestion that the absence of an anti-remodelling effect of sildenafil on pulmonary vessels observed in our study could be attributed to the experimental conditions, since there are no previous studies conducted in guinea-pigs using sildenafil or using CS exposure as a mechanism of pulmonary vascular damage. It is conceivable that the effect of CS exposure on pulmonary vessel structure could exceed those produced by hypoxia, which has been used in previous experimental settings. In fact, in a previous study conducted in guinea-pigs we showed differences in the characteristics of pulmonary vascular remodelling between CS exposure and hypoxia [23]. 
These current observations of sildenafil in the guinea-pig chronically exposed to CS contrast with the effects recently found in this experimental model using a sGC stimulator (BAY 41-2272) [18]. With the latter compound we showed not only reduced pulmonary vascular resistance, but also less pulmonary vascular remodelling. Although no direct comparisons between the two drugs have been made, it is tempting to speculate that sGC stimulation might be more effective in increasing cGMP levels than PDE5 inhibition [28], thereby resulting in a more potent anti-remodelling effect.

Interestingly, the airspace size was lower and the small vessel density was higher in CS-exposed animals treated with sildenafil than in CS-exposed untreated animals and did not differ from nonexposed guinea-pigs. Since the airspace size and the small intrapulmonary vessel density were significantly correlated, our data suggest that sildenafil contributed to the preservation of the structural integrity of the lung. In the present study, changes in airspace size were of small magnitude and therefore insufficient to produce any effect on respiratory resistance or gas exchange, akin to previous observations [18]. The preservation of the intrapulmonary vascular surface, which allows reduced vascular resistance, might explain the reduced PAP in sildenafil-treated animals despite the lack of change in vessel remodelling. The inverse relationship between small-vessel density and RV hypertrophy also points in that direction, although we cannot disregard a direct effect of sildenafil on RV itself as a mechanism of reduced hypertrophy $[24,29]$. We have observed similar effects employing an sGC stimulator, which prevented both pulmonary vascular remodelling and emphysema development in CS-exposed guinea-pigs [18]. This suggests that cGMP plays a key role in preserving lung structure. The mechanisms underlying such effects of sildenafil were not explored in the present study, but the previous study described above showed that increased production of cGMP by sGC stimulation increases mediators of vascular integrity and lung maintenance, such as vascular endothelial growth factor A and fibroblast growth factor 10, and antioxidant enzymes, such as superoxide dismutase 1 , and reduces inflammation by preventing the activation and adherence of circulating inflammatory cells [18]. Accordingly, we hypothesise that the prevention of cGMP degradation by PDE5 inhibition with sildenafil contributed to preserve lung parenchyma and vascular integrity by similar mechanisms, namely antioxidant and anti-inflammatory effects, and the upregulation of mediators of vessel integrity. The fact that sGC stimulation exerts a greater effect on cGMP intracellular levels than the prevention of its degradation by the inhibition of PDE5 [28] might explain why, in the current investigation, the effects of sildenafil on vessel remodelling and airspace size were less pronounced than those observed with the sGC stimulator BAY 41-2272 [18]. There is evidence for a causative role of inducible nitric oxide synthase and peroxinitrite in CS-induced emphysema and vessel remodelling [30]. The effects of peroxinitrite that act in part by oxidising sGC can also be prevented by increasing cGMP levels [18].

The study has some limitations. First, we used a preventive experimental design by starting sildenafil administration at the same time as CS exposure. Accordingly, we cannot state that sildenafil will exert similar effects once the structural changes induced by CS have already developed. Indeed, in patients with severe COPD and mild-to-moderate PH, sildenafil failed to improve exercise tolerance [15]. Second, we used a sildenafil dose similar to that approved in humans to treat pulmonary arterial hypertension [26]. Therefore, we cannot disregard that higher doses could have produced a greater impact on vessel remodelling and/or airspace size. Third, our data suggest that sildenafil contributed to preserve the structural integrity of the lung parenchyma, although whether this is reflected in reduced compliance remains to be established. Finally, assessment of exercise function would have been informative to elucidate whether the effects of sildenafil might translate to patients with COPD. However, the effects of CS exposure on exercise tolerance in the guinea-pig have not been documented and the inclusion of an exercise arm would have increased the complexity of our study.

In conclusion, the results of the present investigation show that sildenafil prevents the development of $\mathrm{PH}$ and RV hypertrophy in an experimental model of COPD induced by chronic exposure to CS. These effects are probably due to a vasodilator effect, along with the preservation of parenchymal integrity and vascular surface, since no effects on vessel remodelling were observed. Current results reinforce the notion that the nitric oxide-cGMP axis is perturbed by CS exposure and clarify the effects of sildenafil in COPD-associated PH. Further investigations are needed to determine the role of PDE5 inhibition once the disease is established.

\section{Acknowledgements}

The authors would like to thank the personnel of the Dept of Pathology at the Hospital Clínic and the animal housing facilities of the University of Barcelona, Barcelona, Spain.

\section{References}

1 Thabut G, Dauriat G, Stern JB, et al. Pulmonary hemodynamics in advanced COPD candidates for lung volume reduction surgery or lung transplantation. Chest 2005; 127: 1531-1536. 
Santos S, Peinado VI, Ramirez J, et al. Enhanced expression of vascular endothelial growth factor in pulmonary arteries of smokers and patients with moderate chronic obstructive pulmonary disease. Am J Respir Crit Care Med 2003; 167: 1250-1256.

3 Peinado VI, Barberà JA, Abate $\mathrm{P}$, et al. Inflammatory reaction in pulmonary muscular arteries of patients with mild chronic obstructive pulmonary disease. Am J Respir Crit Care Med 1999; 159: 1605-1611.

4 Barberà JA, Peinado VI, Santos S, et al. Reduced expression of endothelial nitric oxide synthase in pulmonary arteries of smokers. Am J Respir Crit Care Med 2001; 164: 709-713.

5 Peinado VI, Barberà JA, Ramirez J, et al. Endothelial dysfunction in pulmonary arteries of patients with mild COPD. Am J Physiol 1998; 274: L908-L913.

6 Schermuly RT, Stasch JP, Pullamsetti SS, et al. Expression and function of soluble guanylate cyclase in pulmonary arterial hypertension. Eur Respir J 2008; 32: 881-891.

$7 \quad$ Klinger JR, Abman SH, Gladwin MT. Nitric oxide deficiency and endothelial dysfunction in pulmonary arterial hypertension. Am I Respir Crit Care Med 2013; 188: 639-646.

8 Jeremy JY, Rowe D, Emsley AM, et al. Nitric oxide and the proliferation of vascular smooth muscle cells. Cardiovasc Res 1999; 43: 580-594.

9 Ghofrani HA, Voswinckel R, Reichenberger F, et al. Differences in hemodynamic and oxygenation responses to three different phosphodiesterase-5 inhibitors in patients with pulmonary arterial hypertension: a randomized prospective study. J Am Coll Cardiol 2004; 44: 1488-1496.

10 Sebkhi A, Strange JW, Phillips SC, et al. Phosphodiesterase type 5 as a target for the treatment of hypoxia-induced pulmonary hypertension. Circulation 2003; 107: 3230-3235.

11 Schermuly RT, Kreisselmeier KP, Ghofrani HA, et al. Chronic sildenafil treatment inhibits monocrotaline-induced pulmonary hypertension in rats. Am J Respir Crit Care Med 2004; 169: 39-45.

12 Schäfer S, Ellinghaus $\mathrm{P}$, Janssen W, et al. Chronic inhibition of phosphodiesterase 5 does not prevent pressure-overload-induced right-ventricular remodelling. Cardiovasc Res 2009; 82: 30-39.

13 Galiè N, Corris PA, Frost A, et al. Updated treatment algorithm of pulmonary arterial hypertension. J Am Coll Cardiol 2013; 62: Suppl. 25, D60-D72.

14 Blanco I, Gimeno E, Munoz PA, et al. Hemodynamic and gas exchange effects of sildenafil in patients with chronic obstructive pulmonary disease and pulmonary hypertension. Am J Respir Crit Care Med 2010; 181: 270-278.

15 Blanco I, Santos S, Gea J, et al. Sildenafil to improve respiratory rehabilitation outcomes in COPD: a controlled trial. Eur Respir J 2013; 42: 982-992.

16 Wright JL, Churg A. A model of tobacco smoke-induced airflow obstruction in the guinea pig. Chest 2002; 121: Suppl. 15, 188S-191S

17 Ferrer E, Peinado VI, Díez M, et al. Effects of cigarette smoke on endothelial function of pulmonary arteries in the guinea pig. Respir Res 2009; 10: 76.

18 Weissmann N, Lobo B, Pichl A, et al. Stimulation of soluble guanylate cyclase prevents cigarette smoke-induced pulmonary hypertension and emphysema. Am J Respir Crit Care Med 2014; 189: 1359-1373.

19 Domínguez-Fandos D, Ferrer E, Puig-Pey R, et al. Effects of aclidinium bromide in a cigarette smoke-exposed guinea pig model of chronic obstructive pulmonary disease. Am J Respir Cell Mol Biol 2014; 50: 337-346.

20 Lomask M. Further exploration of the Penh parameter. Exp Toxicol Pathol 2006; 57: Suppl. 2, 13-20.

21 Livak KJ, Schmittgen TD. Analysis of relative gene expression data using real-time quantitative PCR and the $2^{-\Delta \Delta \mathrm{C}_{\mathrm{T}}}$ method. Methods 2001; 25: 402-408.

22 Wright JL, Tai H, Churg A. Vasoactive mediators and pulmonary hypertension after cigarette smoke exposure in the guinea pig. J Appl Physiol 2006; 100: 672-678.

23 Ferrer E, Peinado VI, Castañeda J, et al. Effects of cigarette smoke and hypoxia on pulmonary circulation in the guinea pig. Eur Respir J 2011; 38: 617-627.

24 Weissmann N, Gerigk B, Kocer O, et al. Hypoxia-induced pulmonary hypertension: different impact of iloprost, sildenafil, and nitric oxide. Respir Med 2007; 101: 2125-2132.

25 Zhao L, Mason NA, Morrell NW, et al. Sildenafil inhibits hypoxia-induced pulmonary hypertension. Circulation 2001; 104: 424-428.

26 Galiè N, Rubin LJ, Simonneau G. Phosphodiesterase inhibitors for pulmonary hypertension. $N$ Engl J Med 2010; 362: 559-560.

27 Preston IR, Hill NS, Gambardella LS, et al. Synergistic effects of ANP and sildenafil on cGMP levels and amelioration of acute hypoxic pulmonary hypertension. Exp Biol Med 2004; 229: 920-925.

28 Stasch JP, Evgenov OV. Soluble guanylate cyclase stimulators in pulmonary hypertension. Handb Exp Pharmacol 2013; 218: 279-313

29 Nagendran J, Archer SL, Soliman D, et al. Phosphodiesterase type 5 is highly expressed in the hypertrophied human right ventricle, and acute inhibition of phosphodiesterase type 5 improves contractility. Circulation 2007; 116: $238-248$.

30 Seimetz M, Parajuli N, Pichl A, et al. Inducible NOS inhibition reverses tobacco-smoke-induced emphysema and pulmonary hypertension in mice. Cell 2011; 147: 293-305. 